in den anderen Gewichtsklassen, was auf das niedrige Bewegungsniveau von $761 \mathrm{kcal} /$ Woche zurückgeführt wurde. Der $\mathrm{HbA}_{1 \mathrm{c}^{-}}$ Wert sank in allen Gruppen ähnlich um 0,7 Prozentpunkte.

II Kommentar: Die Daten der Look-AHEADStudie zeigen, dass eine Verhaltensmodifikation hinsichtlich Ernährung und Bewegung das Gewicht erheblich reduzieren kann, auch bei älteren Typ-2-Diabetikern. Die bisherige Datenlage zeigt, dass man von Änderungen des Gewichts nicht unbedingt auf Änderungen im Risikoprofil schließen kann. Studien mit einem Vergleich konservativer versus operativer Intervention belegen in der Regel, dass bariatrische Operationen zwar das Gewicht deutlicher vermindern als eine konservative Therapie, die Risikofaktoren werden jedoch in ähnlichem Maß gebessert. Der Vergleich mit chirurgischen Therapieverfahren ist aktuell, wird doch zurzeit in Deutschland immer häufiger gefordert, Diabetiker auch mit einem BMI $<35 \mathrm{~kg} /$ $\mathrm{m}^{2} \mathrm{zu}$ operieren (metabolische Chirurgie), was gemäß der Leitlinie nur im Rahmen von Studien möglich ist.

In der vorliegenden Studie wurde der $\mathrm{HbA}_{1 c}$ um 0,7 Prozentpunkte reduziert. Das ist ein Ausmaß, das auch durch gängige orale Antidiabetika erzielt wird. Gleichzeitig senkte die Gewichtsabnahme von 11 kg auch den Blutdruck wie ein Antihypertensivum, verminderte die Triglyzeride deutlicher als ein Statin und erhöhte das HDL-Cholesterin wie die Nikotinsäure.

Die Studie lässt den Schluss zu, dass eine Lebensstiländerung Typ-2-Diabetikern grundsätzlich empfohlen werden kann nicht nur zur Gewichtsreduktion, sondern auch zur Besserung des gesamten Risikoprofils.

Prof. Dr. med. Alfred Wirth, Bad Rothenfelde II

II Unick JL et al. Effectiveness of lifestyle interventions for individuals with severe obesity and type 2 diabetes. Diabetes Care 2011;34:2152-2157

\title{
Framingham-Studie
}

\section{Fettniere korreliert mit Hypertonie und Niereninsuffizienz}

\section{Welche Folgen Fettablagerungen an einer bislang kaum beachteten Stelle, dem Nierensinus, haben, wurde in der vorliegenden Studie untersucht.}

Die Untersuchungen fanden an 2923 Teilnehmern der Framingham-Studie statt. Das Fett im Nierensinus wurde computertomografisch quantifiziert. Die Definition einer Fettniere basierte auf der 90\%-Perzentile des Nierensinusfetts eines gesunden Referenzkollektivs. Mithilfe multivariater Regressionsanalysen wurde die Korrelation des Nieren-

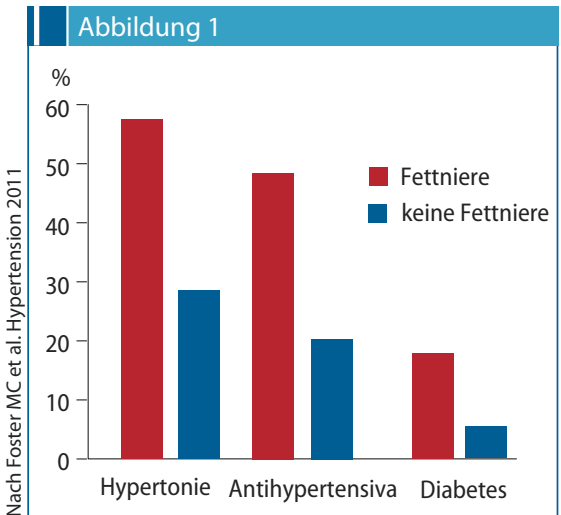

Abb. 1 Häufigkeit von Hypertonie, Antihypertensivagebrauch und Diabetes mellitus in Abhängigkeit von der Existenz einer Fettniere (definiert durch das Nierensinus-Fett oberhalb der 90\%-Perzentile gesunder Menschen. sinusfetts mit metabolischen und vaskulären Parametern untersucht.

Abbildung 1 zeigt die jeweilige Häufigkeit von Hypertonie, Anwendung von Antihypertensiva und Diabetes mellitus in den Gruppen mit und ohne Fettniere. Die jeweiligen Unterschiede in der Prävalenz sind hochsignifikant. Aus diesem Befund ergibt sich die weitere Frage, ob das Nierensinusfett auch dann noch ein Prädiktor für eine $\mathrm{Hy}$ pertonie ist, wenn für den Einfluss des viszeralen Fettgewebes eine statistische Adjustierung erfolgt. Naturgemäß korrelierten auch

\section{Abbildung 2}

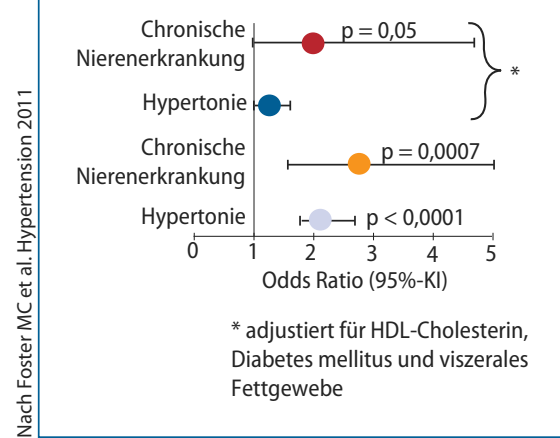

Abb. 2 Risiko für Hypertonie oder chronische Nierenerkrankung in Abhängigkeit von der Existenz einer Fettniere. die Menge des viszeralen Fetts und des Nierensinusfetts miteinander.

Abbildung 2 zeigt die Odds Ratios für das Vorhandensein einer Hypertonie bzw. einer Niereninsuffizienz (entsprechend des Serum-Cystatins). Interessanterweise ist die Menge des Nierensinusfetts auch dann ein signifikanter Prädiktor für Hypertonie und Niereninsuffizienz, wenn statistisch eine Adjustierung für den Einfluss des viszeralen Fettgewebes erfolgt.

II Kommentar: Über die Gründe für den möglichen Einfluss des Nierensinusfetts auf die Entstehung von Hypertonie und Niereninsuffizienz kann nur spekuliert werden. Denkbar ist, dass ein vermehrtes Nierensinusfett den Abfluss aus Nierenvenen und Nierenlymphgefäßen behindert. Dadurch könnte der Druck im interstitiellen Nierengewebe zunehmen und die Kochsalzausscheidung vermindert werden. Dieser Mechanismus kann sowohl einer verminderten Nierenfunktion als auch einem gesteigerten Blutdruck zugrundeliegen.

Prof. Dr. med. Walter Zidek, Medizinische Klinik IV, Charité Berlin II

II Foster MC et al. Fatty Kidney, Hypertension, and Chronic Kidney Disease: The Framingham Heart Study. Hypertension, published online 19. September 2011 